\title{
Bioética de la salud pública*
}

\section{Giovane Mendieta-Izquierdo ${ }^{* *}$ Juan María Cuevas-Silva ${ }^{* * *}$}

La bioética está llamada a realizar un ejercicio comprensivo, que le permita identificar elementos propios de la salud en torno a las dinámicas del ejercicio médico y la salud pública. Es importante, asimismo, que se posicione desde la comprensión teórica, conceptual y empírica del derecho humano a la salud, sin perder de vista que su punto de partida son las realidades sociales, y debe tener en cuenta que mientras tanto avanza el campo de la política en salud, en el marco de la esfera individual (clínica-médica) o colectiva (salud públicacomunitaria).

Desde el concepto de salud pública-comunitaria, se pretende situar a la salud en el centro de la justicia social, sin dejar de lado el contexto y sus dinámicas de implementación de los sistemas bioéticos, abordados desde un análisis del contexto internacional, latinoamericano hasta llegar a la esfera nacional. Es necesario incluir en esta comprensión aspectos propios de la diversidad, diferencia, multiculturalidad, condiciones socioeconómicas y políticas de la atención en salud, en el contexto propio del avance vertiginoso de la ciencia y la tecnología propia de nuestra sociedad, que además se entremezclan con la conciencia y acción moral propias de una sociedad que se encuentra en crisis por la emergencia de neoconservadurismos y relativismos éticos que afectan una hermenéutica de la fenomenología bioética.

\footnotetext{
* Algunos apartados de este artículo hacen parte del documento Línea de Investigación en Bioética Médica y Salud Pública, desarrollado en los Seminarios Núcleo Problémico I y II del Doctorado en Bioética, de la Universidad Militar Nueva Granada.

** Ph.D. en Ciencias de la Salud Pública. Profesor investigador en Bioética y coeditor Revista Latinoamericana de Bioética, de la Universidad Militar Nueva Granada, Bogotá, Colombia. Correo electrónico: giovane.mendieta@unimilitar.edu.co. ORCID: org/0000-0002-5085-3242

*** Candidato a Ph.D. en Procesos Sociales y Políticos de América Latina. Profesor investigador y editor de la Revista Latinoamericana de Bioética, de la Universidad Militar Nueva Granada. Bogotá, Colombia. Correo electrónico: juan.cuevass@unimilitar.edu.co
} 
Así es como la bioética médica-clínica es comprendida como la esfera individual. Esta

se ha preocupado por tener en el centro del debate los aspectos propios de la práctica médica en el marco de los avances tecno-científicos y la relación médico/paciente; desde este punto, los dilemas bioéticos han emergido, han sido abordados y desarrollados. El mayor desarrollo conceptual, reconocido como hegemónico en la bioética médica, se ha dado en analogía a modelos aplicados a esta relación. Así, lo interesante de retomar temas de la bioética médica es precisamente analizarlos desde el contexto social, político y cultural contemporáneo caracterizado por el influjo de paradigmas morales, prácticas sociales y contextos divergentes, que en su interior y esencia son bioéticos, aspecto que permite comprenderlos como emergentes. En este sentido, en Colombia, situaciones particulares con implicaciones bioéticas como la implementación de la Ley 1805 de 2016 en la que se regula la donación de órganos, o la gestación por sustitución y fertilización in vitro a la luz del concepto de familia son algunos de los temas vigentes en el análisis de la bioética médica colombiana, que exigen un abordaje investigativo bioético, sin omitir los microcontextos y macrocontextos sociales, culturales, morales, religiosos, seculares, económicos, emocionales.

Por otro lado, la salud pública, comprendida como la esfera colectiva, invita a formular la pregunta: ¿es necesario un análisis bioético que permita la comprensión de prácticas sociales, culturales o locales en torno a la salud pública? La salud pública en sí es un aspecto emergente en el contexto de la bioética, ya que en las distintas tendencias investigativas de la bioética ha sido soslayada o sencillamente se deja como algo implícito, cuando realmente debe ser un problema de investigación bioética que amerita ser explicado.

La naciente literatura sobre bioética de la salud pública ha alcanzado los debates más allá de los problemas centrados en la relación médico-paciente, de tal forma que en la bioética de la salud pública están pendientes investigaciones, estudios y abordajes sobre las causas sociales de la salud en las poblaciones, la corrupción estructural de la política estatal con el manejo de recursos para la salud pública, la desconexión de esta última y la formación del profesional de la salud (enmarcada aún en un modelo curativo), la mercantilización y estratificación de la salud (una para ricos, otra para pobres), entre otros aspectos. Desde la salud pública, la bioética se ha contextualizado desde los lineamientos de la Organización Mundial de la Salud (OMS) con la iniciativa Ética y Salud (2002), que orienta las políticas públicas del sector 
salud. En Latinoamérica, y en particular en Colombia, se destacan los compromisos adquiridos en la Iniciativa de Salud de las Américas, que incluye el desarrollo de las funciones esenciales de salud pública, la agenda de acuerdos entre los ministros de salud de la región andina, los Objetivos de Desarrollo del Milenio y el Reglamento Sanitario Internacional, así como otros compromisos internacionales en temas específicos que convergen en el Plan Decenal de Salud Pública (PDSP) (2012). Los aspectos mencionados sirven de base para la reflexión en torno a la importancia social de las políticas y los propios alcances de la acción, a partir de las bases conceptuales del saber propio de la salud pública y su relación con la bioética.

En este orden de ideas, las decisiones que se toman en salud pública en torno a la asistencia sanitaria, planificación, atención clínica y biotecnológica se deben enmarcar desde una perspectiva bioética teniendo en cuenta elementos conceptuales que decantan en varios enfoques: diferencial, ciclo de vida, género, étnico y poblacional, entre otros, analizados siempre desde la perspectiva de derechos humanos, determinantes sociales (CSDH) y justicia social en salud, aspecto que sugiere la comprensión, reflexión y postura frente a ellos (PDSP, 2012). En ese sentido, la bioética representa un soporte importante para orientar las decisiones en salud pública, de tal manera que las elucubraciones bioéticas basadas en moralismos o relativismos pasan a ser agentes articuladores entre la realidad social, cultural, política y económica, y la salud pública con integridad y sentido bioético.

Por otra parte, la Declaración Universal sobre Bioética y Derechos Humanos, expedida por la Organización de las Naciones Unidas para la Educación (Unesco) (2005), es uno de los marcos referenciales para establecer los lineamientos que en materia de bioética se requiere incorporar en la toma de decisiones, acciones de intervención y políticas estratégicas en salud pública. Desde este marco, los profesionales de la salud (concebidos como salubristas públicos), en general, y el bioeticista, en particular, deben analizar situaciones respecto a la dignidad humana, los derechos humanos, las libertades individuales, el derecho a ejercer la ciudadanía desde la igualdad, equidad y conciencia colectiva, desmarcándose de los procesos de mercantilización de la salud, así como de sus dogmatismos (incluido el relativismo dogmático) y modelos mentales que limitan y castran la comprensión de otras realidades 
humanas y sociales en las que se desvanecen los discursos de la tolerancia, "el amor al prójimo”, el espíritu de servicio y la vocación bioética del profesional de la salud pública.

Lo anterior se fundamenta a la luz de la Declaración Universal sobre Bioética y Derechos Humanos (Unesco, 2005) cuando se introduce una mirada holística de la bioética (donde está implícita la salud pública), no antropocéntrica, pues incluye todo lo que es vida y que tiene estrecha relación con la salud humana, animal, biótica y no biótica. Esto en un contexto nostálgico por el bienestar de lo ecológico y del medio ambiente, donde la salud no se comprende solamente desde un paradigma mecanicista antropocéntrico, y que invita a desarrollar enfoques nuevos en torno a la responsabilidad social en salud, la justicia y equidad, alejado de toda forma de estigma, discriminación e intolerancia; además, apostándole a la comprensión e inclusión de la diversidad cultural, promoción de la salud y protección del medio ambiente como responsabilidad imperativa de los Gobiernos desde lo nacional, regional y local, que deben fomentar estratégica y contextualmente espacios de evaluación y gestión del riesgo desde la salud, ciencias de la vida y tecnología (PDSP, 2012).

La bioética en salud pública debe hacer hincapié en la comprensión y discusión desde la vinculación de los problemas y dilemas que plantea el contexto de la salud pública, y así intentar vincular en un marco de conciencia moral y acción ética el empoderamiento en la salud de las poblaciones, así como la vinculación de esta en la formulación de políticas públicas sanitarias, donde se pretenda generar conciencia en los procesos de cambio de las prácticas sociales para brindar elementos de comprensión a una bioética socialmente contextualizada. El debate desde la OMS gira en torno a los determinantes sociales (CSDH), aspecto que genera tensión a nivel latinoamericano con la emergente discusión en torno al determinismo y la determinación social. Así es como el debate determinantes (CSDH)determinismo-determinación social-indeterminación (Breilh, 2013; Peñaranda y Rendón, 2013) sitúa a la salud pública en el centro de la justicia social (Venkatapuran y Marmot, 2009), y da paso a la emergente categoría justicia social en salud (Venkatapuran, 2013), primordial para el desarrollo de la bioética de la salud pública.

\section{Referencias}


Breilh J. (2013). La determinación social de la salud como herramienta de transformación hacia una nueva salud pública (salud colectiva). Revista Facultad Nacional de Salud Pública, 31(supl. 1), S13-S27.

Colombia, Congreso de la República (4 de agosto de 2016). "Ley 1805 de 2016”. Bogotá, Colombia. Recuperado el 7 de junio de 2017, de http://es.presidencia.gov.co/normativa/normativa/LEY\%201805\%20DEL\%2004\%20DE\%2 0AGOSTO\%20DE\%202016.pdf

Declaración Universal sobre Bioética y Derechos Humanos. (19 de octubre de 2005). Unesco. Recuperado el 7 de junio de 2017, de http://portal.unesco.org/es/ev.phpURL_ID=31058\&URL_DO=DO_TOPIC\&URL_SECTION=201.html

Peñaranda F. y Rendón, C.E. (2013). Determinismo-indeterminismo y el debate de los determinantes-determinación social de la salud. Revista Facultad Nacional de Salud Pública, 31(supl. 1), S47-S56.

Ministerio de Salud y Protección Social. (2012). Plan Decenal de Salud Pública 2012-2021. $\begin{array}{lllllll}\text { Recuperado el } & 7 & \text { de } & \text { junio }\end{array}$ https://www.minsalud.gov.co/Documentos\%20y\%20Publicaciones/Plan\%20Decenal\%20$\% 20$ Documento\%20en\%20consulta\%20para\%20aprobaci\%C3\%B3n.pdf

Venkatapuran, S. (2013). Health, vital goals, and central human capabilities. Bioethics, 27(5), 271-279. doi:10.1111/j.1467-8519.2011.01953.x

Venkatapuran, S. y Marmot, M. (2009). Epidemiology and Social Justice in Light of Social Determinants of Health Research. Bioethics, 23(2), 79-89. doi:10.1111/j.14678519.2008.00714.x 